\title{
QU'EST-CE QU'UNE RESSOURCE LOCALE? HOMÉOSTASIE ET MICROANALYSE EN HISTOIRE SOCIALE
}

\author{
Paul-André Rosental
}

Résumé: L'historiographie française prête une attention croissante à l'échelle microscopique, en associant essentiellement cette dernière à la microstoria italienne. Il existe pourtant, au Royaume-Uni et en Allemagne notamment, d'autres traditions nationales de «microhistoire », qui proposent des usages différents de ce niveau d'observation. De même, les propriétés prêtées au micro (le primat du local, le refus des explications fonctionnalistes) ne lui sont pas nécessairement spécifiques. À partir d'un objet précis, la question du rapport entre populations et ressources et sa formulation par le modèle dit «homéostatique », l'article s'efforce de nuancer l'idée selon laquelle chaque échelle serait dotée d'attributs qui lui seraient propres. En montrant les convergences possibles entre des niveaux d'observation apparemment incommensurables, il considère que les effets d'échelles dépendent davantage de situations historiographiques locales que de propriétés abstraites.

Mots-CLÉs : microhistoire, historiographie, local, sources, modèle homéostatique.

ABSTRACT: French historiography is giving increasing attention to the microscopic scale by associating it essentially with the Italian microstoria. There are nevertheless in the United Kingdom and in Germany notably, other national traditions of "microhistory", which propose different uses of this level of observation. Similarly, the properties ascribed to the micro (the primacy of the local dynamics, the refusal of functionalist explanations) are not necessarily specific to it. Beginning with a precise issue the question of the relationship between populations and resources and its formulation by the model called "homeostatic", the article strives to nuance the idea according to which each scale would be granted its own attributes. By showing the possible convergences between apparently incommensurable levels of observation, it considers that the effects of scales depend more on local historiographic situations than on abstract properties.

KEYWORDS : microhistory, historiography, local, sources, homeostatic model. 
Zusammenfassung : Die französische Geschichtsschreibung widmet sich in zunehmendem Maße den Mikrostrukturen zu, wobei sie im wesentlichen an der italienischen microstoria anknüpft. Es gibt jedoch in Großbritannien und in Deutschland noch andere nationale Traditionen einer "Mikrogeschichte», bei denen ein anderes Vorgehen auf diesem Niveau der Beobachtung vorgeschlagen wird. Die dem «Mikroniveau» zugeschriebenen Merkmale (Primat des Lokalen, Ablehnung funktionaler Erklärungen) sind dabei keineswegs das Entscheidende. Ausgehend von einem genau definierten Gegenstand, nämlich der Frage nach den Beziehungen zwischen Populationen und Ressourcen und der Beschreibung dieses Themas mit dem sogenannten homöostatischen Modell, wird in diesem Artikel der Versuch unternommen, die Vorstellung zu hinterfragen, daß jeder Maßstab seine eigenen Attribute besitzt. Indem mögliche Konvergenzen zwischen Beobachtungsniveaus aufgezeigt werden, die scheinbar imkommensurabel sind, wird der Standpunkt vertreten, daß Maßstabseffekte mehr von lokalen historiographischen Situationen als von abstrakten Merkmalen abhängen.

STICHWÖRTER: Mikrogeschichte, Geschichtsschreibung, örtlicher Bezug, homöostatisches Modell.

RIASSUNTO: La storiografia francese presta un'attenzione crescente alla scala microscopica, associando essenzialmente quest'ultima alla microstoria italiana. Esistono tuttavia, particolarmente in Gran Bretagna e Germania, altre tradizioni nazionali di microstoria, che propongono degli usi diversi di questo livello di osservazione. In aggiunta, le proprietà attribuite al micro (il primato del locale, il rifiuto delle spiegazioni funzionaliste) non sono necessariamente specifiche di quest'ultimo. A partire da un oggetto preciso - la questione del rapporto tra popolazione e risorse e la sua formulazione nel modello detto "omeostatico » - l'articolo cerca di sfumare l'idea secondo la quale ogni scala sarebbe fornita di attributi che gli sarebbero propri. Mostrando le convergenze possibili tra livelli di osservazione apparentemente incommensurabili, considera che gli effetti di scala dipendono più da situazioni storiografiche locali che da proprietà astratte.

Parole-CHIAVE : microstoria, storiografia, risorse locali, modello omeostatico.

Paul-André RosenTAL, né en 1961, est maître de conférences à l'École des hautes études en sciences sociales et chercheur associé à l'Institut national d'études démographiques. Son travail porte sur l'histoire sociale des migrations et de la famille, et sur l'histoire des sciences sociales.

Adresse: EHESS, Centre de recherches historiques, 54 bd Raspail, F-75006 Paris.

Courrier électronique : rosental@ehess.fr 
L'analyse microhistorique a fait l'objet d'une attention croissante en France depuis une quinzaine d'années. Importée d'Italie sous l'effet des nombreux contacts institutionnels et personnels entre les deux pays, en premier lieu mais pas exclusivement autour de l'École des hautes études en sciences sociales et des anciens de l'École de Rome, elle a été reçue comme une approche révolutionnaire, chamboulant les habitudes acquises dans les années soixante et soixante-dix sous l'influence de l' «école des Annales ». Le passage du « macro » au «micro »a déplacé l'attention des grandes déterminations économiques (modèle labroussien) ou «culturelles » (histoire des mentalités), à l'observation des parcours individuels et des configurations interpersonnelles retracées dans leur complexité. Il a aussi remis en cause la notion de contexte, perçu non plus comme un cadre extérieur et antérieur à l'analyse, mais comme une réalité dynamique, relative à chaque situation locale et reconstructible, de manière connexionniste $^{1}$, à partir d'un microévénement anodin ${ }^{2}$. Au total, ce déplacement a produit deux types d'espérances, formulées autour de 1990 dans les Annales $^{3}$. Pour les uns, les niveaux d'observation se valent mais sont plus ou moins discontinus ${ }^{4}$ : le micro présente un intérêt contingent, historique, celui de faire changer d'échelle, son aspect d'estrangement ${ }^{5}$ produisant une connaissance neuve. Pour les autres, le micro constitue la bonne échelle d'observation du social, y compris pour l'explication des processus agrégés. À l'extrême, on devrait parler de «microhistoire » comme on parle de « microéconomie ».

Parallèlement à ces débats théoriques, qui atteignent leur apogée entre la fin des années quatre-vingt et le milieu des années quatre-vingt-dix ${ }^{6}$, une espèce de «sensibilité » microscopique se diffuse largement. Fort éloignée de la microstoria mais pas de l'une de ses compagnes de route, l'histoire orale des années soixante-dix, elle s'en réclame pour légitimer des choix d'objets réduits, et refonder l'intérêt croissant des historiens pour l'intime,

1. Nous empruntons volontairement cette expression à la sociologie latourienne, dont la construction (au moins dans ses débuts) n'est pas sans analogie à celle de la microstoria. Loin d'être le fait du hasard, la parenté repose sur leur commune racine ethnographique.

2. On trouvera, en France, une illustration de la méthode in FARGE et Revel, 1988, qui, à partir d'un mouvement de foule du milieu du XvIII ${ }^{\mathrm{e}}$ siècle, débouchent sur une analyse de la légitimité du pouvoir royal.

3. «Tentons l'expérience », 1989.

4. Selon une formulation bien explicitée par LePETIT, 1993, qui s'appuie entre autres sur des références aux sciences de la nature. Sur l'incommensurabilité d'échelles en physique, voir SCHWEBER, 1993.

5. Milo, 1991.

6. Revel, éd., 1996. 
l'individuel et le contingent. La distance avec les auteurs italiens est pourtant évidente : alors que ceux-ci s'efforcent de reconstruire la société sur la base d'observations micro, il s'agit surtout ici de montrer en quoi des parcours singuliers incarnent des processus plus larges, de donner de la chair à des scénarios historiques abstraits qui ne sont pas remis en cause par l'analyse. Le micro sert d'illustration, permettant à l'historien, dans ses réalisations les plus abouties, de déployer son savoir sous une forme plus humaine ${ }^{7}$.

Quelle que soit leur diversité, ces discussions s'accordent sur un scénario comparable $^{8}$ : la microhistoire est originellement une sensibilité italienne, son introduction en France correspond à un déplacement des intérêts des historiens (ainsi que de leur mode de narration et de démonstration) et produit, sinon une rupture, du moins une forte inflexion historiographique. Cette lecture décrit fort bien ce qu'on pourrait appeler trivialement la sortie de la période « Nouvelle Histoire », c'est-à-dire la remise en cause des approches (souvent françaises) qui avaient dominé l'historiographie dans les années soixante et soixante-dix ${ }^{9}$. Elle vaut pour une période, et pour un type de transfert, italo-français, même si celui-ci, par ricochet, commence à toucher le monde américain. Mais d'un point de vue tant historiographique que théorique, elle est loin d'épuiser la question de l'usage du « micro » en histoire.

À un discours historiographique général mais ponctuel, l'objet de cet article est d'opposer une analyse qui suive l'usage contemporain de l'échelle «micro» dans un domaine de la connaissance historique, d'observer ses apports mais aussi de discuter ses limites. L'objet que nous avons retenu à cet effet est le modèle dit «homéostatique », qui s'efforce de formaliser les relations entre populations et ressources dans un environnement donné, et en particulier les processus de récupération démographique après une crise. Il fournit également un cadre théorique aux explications «négatives» des migrations, qui voient dans ces dernières l'éviction d'individus en surnombre par rapport aux ressources locales.

La raison de ce choix est multiple. Tout d'abord, plutôt que de surimposer une problématique, il permet de saisir un débat historiographique en cours explicitement formulé en termes d'échelles : la question de savoir si le modèle homéostatique peut résister aux conclusions des études microhistoriques fait, de nos jours, l'objet de discussions internationales au sein

7. L'ouvrage de CoRBIN, 1998, et son succès d'édition, en sont une illustration exemplaire, et sans doute l'une des plus riches. Voir aussi, dans le monde anglo-saxon, le travail de ScotT, à paraître. Dans d'autres cas, l'auto-étiquetage « microhistorique » masque tout bonnement un retour à la monographie.

8. ReVEL, 1989.

9. Les critiques de Stone, 1980, ont joué un rôle important dans ces débats, que retrace ReVel, 1989. 
des historiens démographes ou économistes ${ }^{10}$. En deuxième lieu, le modèle homéostatique a une histoire longue, où l'échelle d'observation a toujours occupé une place décisive ${ }^{11}$. Elle chevauche plusieurs pays et mobilise de nombreuses écoles microhistoriques nationales, dont certaines ont nourri la microstoria. Sans avoir ici à la retracer, son évocation nous permettra de faire référence à des usages différents de l'échelle microscopique. Enfin, l'un des intérêts du modèle homéostatique est de soulever la question du local à travers une approche plus formalisée que d'ordinaire en histoire. On peut ainsi briser l'équation consubstantielle entre «micro » et « local » et montrer que ces notions se remettent au contraire mutuellement en question.

\section{UN MODÈLE PLASTIQUE}

Les questions que nous venons de soulever concernent la recherche contemporaine en histoire sociale et en démographie, où se mêlent indissolublement production de savoir nouveau et réflexion sur l'histoire de l'outil $^{12}$. D'origine récente (en histoire, il est explicitement utilisé comme tel depuis le milieu des années soixante ${ }^{13}$ ), le modèle homéostatique sert à formaliser les variations de populations en longue période, ou les réactions de court terme à une crise. Sa caractéristique est de modéliser les processus de stabilisation, ou plus précisément d' «auto-régulation » démographiques. Plutôt que d'insister sur la mortalité, il met l'accent sur le rôle de la nuptialité : dans les sociétés, avant tout rurales, dans lesquelles le nombre de «places » est supposé limité, la reproduction est conditionnée à l'accès à une forme d'autonomie économique et, en premier lieu, à la prise en charge d'une exploitation. Une partie de la population est ainsi socialement stérilisée ${ }^{14}$ ou contrainte à la migration. Son volume fluctue avec la démographie : dans ces espaces économiques inélastiques, la croissance du

10. Elle faisait l'objet de la session « Micro-history and the macro-models of the European economic-demographic system in pre-industrial times » au cours du Congrès international d'histoire économique de Madrid en 1998, selon une problématique définie par Schlumboнm, 1996.

11. Comme le montre Coleman, 1986, p. 14 sq.

12. C'est en ces termes qu'était formulée la session qui s'est récemment tenue lors de la $3^{\text {e }}$ «Social Science History Conference » organisée à Amsterdam en avril 2000, qui réunissait historiens des sciences, historiens et anthropologues autour de l'histoire du modèle homéostatique.

13. Par opposition à d'autres disciplines, où il a commencé à se diffuser dès les années vingt, en partant d'un premier usage en physiologie analysé par LANGLEY, 1973.

14. Il arrive que les terminologies locales entérinent ce processus, appelant par exemple «esterlo » les cadets exclus de la succession. 
nombre d'habitants favorise la crise ou en aggrave les effets. En multipliant les vacances dans les différentes niches économiques, la mortalité augmente les occasions disponibles pour les jeunes adultes. Ceux-ci accèdent plus massivement ou plus précocement à la formation d'un foyer, ce qui relance la fécondité.

Son caractère formalisé, sa capacité à penser des évolutions nonlinéaires, sa correspondance avec les représentations que l'on a des sociétés du passé ou des pays en développement, font du modèle homéostatique un outil fort usité tant en démographie qu'en histoire ${ }^{15}$. Sa relative plasticité est également un atout : selon les terrains et les points de vue, on peut le considérer comme une extension du schéma malthusien ou au contraire comme un raisonnement alternatif, rompant aussi bien avec le catastrophisme du positive check qu'avec le moralisme ou le fonctionnalisme du preventive check. On peut l'adapter à des sociétés industrielles, ou en réserver l'application à certains types de sociétés ${ }^{16}$. En termes d'échelle temporelle, le modèle homéostatique se prête aussi bien à l'analyse des effets de la conjoncture démographique courte qu'à la modélisation d'oscillations démographiques pluriséculaires. Le modèle d' «histoire immobile » proposé par Emmanuel Le Roy Ladurie, emblème des Annales dans les années soixante-dix, en est une application directe : il décrit les oscillations de la population française entre un plancher et un plafond démographiques entre le bas Moyen Âge et le XVIII ${ }^{\mathrm{e}}$ siècle, qu'il transforme du même coup en une période unique.

La même ambiguïté joue sur son échelle. Qu'à compter de la fin des années quatre-vingt, l'analyse microhistorique ait été amenée à remettre en cause le modèle ne signifie pas que celui-ci se situerait par essence au niveau macroscopique. En fait, il a été mis en œuvre dans le passé aussi bien sur des données agrégées nationales mettant en relation croissance démographique et grands indicateurs économiques ${ }^{17}$, que sur des observations recueillies à l'échelle du village, voire de l'exploitation agricole ${ }^{18}$. Aussi, le déplacement proposé par la microanalyse a moins consisté à privilégier l'échelle fine des individus, ménages ou lignées, qu'à aller au-delà de la seule observation des tendances globales pour ne plus attribuer $a$ priori un même type de comportement et de réaction, mécanique, à toute la population étudiée ${ }^{19}$.

L'une des critiques «micro » les plus importantes du modèle a trait à sa territorialité superficielle: tout son mécanisme repose sur l'idée d'un

15. LEE, 1978.

16. LEE, 1990.

17. Wrigley et Schofield, 1981; et les nombreux travaux qui se sont appuyés sur cet ouvrage.

18. DupÂquier, 1972.

19. Fertig, 1998; Bourdieu, Postel-Vinay, Rosental et Suwa-Eisenmann, 2000. 
nombre limité de «places » en un espace donné, sans pour autant le définir ni véritablement s'interroger sur ses bornes. Cet implicite est d'autant plus problématique que les ruralistes ont de longue date mis l'accent sur la capacité des villageois à trouver des ressources complémentaires, non seulement localement par le biais de la pluriactivité, mais aussi ailleurs au moyen de migrations temporaires. Les frontières du local, leur plasticité selon les villages, voire selon les lignées, deviennent dès lors une dimension fondamentale de la recherche : c'est précisément par cet angle que l'on peut approcher la spécificité, les apports, mais aussi les limites, d'un regard microscopique.

Dans son Pouvoir au village, Giovanni Levi illustre, au milieu des années quatre-vingt, le type de déplacement produit par la microstoria ${ }^{20}$. Ses prémisses prennent le contre-pied des théories de la reproduction sociale : elles affirment que même dans un monde à première vue aussi stable qu'un village piémontais de la fin du XVII ${ }^{\mathrm{e}}$ siècle, c'est l'incertitude qui préside à la perpétuation tant intra- qu'intergénérationnelle des positions socio-économiques. Il convient, dès lors, d'éviter les déviations finalistes pour retrouver la difficulté inhérente à toute décision paysanne: acheter ou non une terre disponible, par exemple, au risque soit de perdre une affaire, soit de se retrouver ruiné en cas de malheurs démographiques toujours possibles. Il s'agit pour l'historien de redessiner les contraintes, mais aussi les ressources, des individus du passé : en suivant pas à pas les comportements des paysans, on s'aperçoit qu'ils mutualisent le risque en mobilisant leur parenté étendue, et en créant des «fronts » qui se partagent les charges économiques au travers de contrats formels devant notaires.

La conclusion est elle aussi typique de la microstoria : elle signifie que bien qu'il ait été relayé et légitimé par l'historiographie ${ }^{21}$, le cadre du ménage proposé par les sources habituelles (listes de population) n'est pas pertinent à la saisie de l'action quotidienne. Celle-ci prend sens à une autre échelle, qui n'est pas celle du foyer mais de la famille étendue. La méthode qui a permis ce déplacement est ethnographique (pratiquer une observation intensive en partant d'un postulat d'ignorance) ${ }^{22}$ et relationnelle : les liens

20. LEVI, 1989.

21. Laslett et Wall, éd., 1972.

22. La référence à l'ethnographie n'est pas métaphorique : elle reflète l'intérêt qu'ont porté les microhistoriens transalpins à l'anthropologie sociale, notamment britannique. 
interpersonnels constituent le ressort et les limites de l'action. Invisibles dans les dénombrements démographiques ou fiscaux (qui privilégient le ménage), ils sont observables à travers d'autres sources, notariales par exemple (qui permettent de retrouver les liens de créanciers à débiteurs, ou encore de vendeurs à acheteurs de biens fonciers).

Au-delà de ces considérations immédiates, la remise en cause de la pertinence du ménage comme unité sociale privilégiée débouche sur une relecture de la territorialité des ressources économiques. Le ménage est en effet défini par la corésidence, aux frontières bien circonscrites. Or, les résultats de Levi viennent rappeler que la cohérence de cette équation est moins sociohistorique qu'administrative, et que les historiens de la famille se sont laissé imposer une catégorisation incontrôlée issue des procédures de la bureaucratie (ancienne et contemporaine). Pour la question qui nous concerne, émerge dès lors la nécessité de comprendre où s'arrêtent les limites spatiales pertinentes à l'action sociale et, en particulier, à l'accès aux ressources de l'environnement.

Cette interrogation n'est évidemment pas limitée au Piémont de l'époque moderne. On peut la reprendre et la développer en d'autres terrains, en partant comme Levi d'une réflexion sur les sources. La France rurale du $\mathrm{XIX}^{\mathrm{e}}$ siècle en fournit l'occasion, qui a fait l'objet d'une longue enquête collective fondée sur une entrée généalogique ${ }^{23}$. Ce matériau, rarement disponible à une si grande échelle, peut faire l'objet d'une réflexion intrinsèque : que devient la notion de migration si on l'appréhende non pas à travers des séries statistiques agrégées mais à travers le suivi de trajectoires dynastiques et, plus précisément, en prenant au sérieux la dimension nominative de ce type d'informations? L'observation de parcours à première vue singuliers modifie-t-elle un questionnement dominé par la tradition macroquantitative $^{24}$ ?

La question permet d'expliciter un certain nombre de principes de l'analyse microhistorique : l'observation intensive des configurations et des trajectoires personnelles; le fait de saisir les individus en action, dans la série de leurs comportements successifs, et non à plat, à travers des catégories prédéfinies; une posture de méfiance méthodologique à l'égard des notions ou des outils hérités du passé — cadre de classement des sources, catégories historiographiques. Leur mise en œuvre conduit à une espèce de déconstruction pratique. Sans se substituer à une histoire des sciences sociales ou des procédures administratives, elle permet, par la transposition

23. L'enquête collective dite Trois mille familles s'est efforcée, durant les années quatrevingt, de reconstituer les parcours généalogiques de 3000 lignées présentes sur le territoire français à partir du début du $\mathrm{XIX}^{\mathrm{e}}$ siècle. Voir sa présentation in DUPÂQUIER et KESSLER, éd., 1992.

24. Sur ces développements et ceux qui suivent, voir Rosental, 1999. 
en histoire de l'observation de terrain, de reconstituer des logiques jusqu'alors inaperçues.

En effet, le glissement vers l'échelle familiale fait apercevoir des mobilités complexes dans un territoire familier, dont les bornes sont données par la localisation des proches (parents ou non-parents). La définition classique de la migration comme un changement de résidence et l'opposition qui en découle entre un point de départ et un point d'arrivée s'y trouvent relativisées au profit d'un raisonnement en termes de circulation dans un univers familier - lequel peut inclure des destinations géographiquement éloignées. Le décalage archivistique met en évidence une détermination cognitive produite par l'histoire : c'est le cadre de la statistique administrative, l'opposition qu'il a tracée entre points d'origine et d'arrivée des flux, qui a structuré notre perception même de la migration aux dépens de toute autre saisie du phénomène ${ }^{25}$. Ses répercussions historiographiques sont immédiates, et nullement cantonnées au niveau micro : l'observation permet de donner sens à l'intense mobilité rurale ${ }^{26}$, mal connue dans sa nature et ses effets, et volontiers négligée dans son importance ${ }^{27}$.

Mais l'adoption d'une méthode microscopique d'analyse ne se borne pas à définir une nouvelle unité sociale pertinente. Substituer au ménage la parenté élargie ne signifie pas que cette dernière opère de manière univoque, ni même qu'elle soit toujours déterminante. En saisissant les lignées dans leur dimension diachronique, en insistant sur leurs temporalités propres, on observe qu'elles mettent en œuvre des fonctionnements différents, inégalement centrés sur la famille. À chacun correspond une gamme spécifique d'engendrement des migrations : on ne se déplace pas de la même manière, ni pour les mêmes raisons, selon que l'on appartient à une forme ou à une autre de lignée ${ }^{28}$.

Cette perspective micro n'en aboutit pas pour autant au constat banal, et pour partie relativiste, de la diversité et de la complexité des formes

25. L'anthropologie est fréquemment placée dans une situation comparable, comme en témoigne The Territorial Mobility of population. Rethinking its forms and functions, 1979.

26. Pour le $\mathrm{XIX}^{\mathrm{e}}$ siècle, elle est près de dix fois supérieure aux migrations vers les villes qui sont d'ordinaire les plus visibles, et elle dépasse même en fréquence les cas de sédentarité communale.

27. Tout en l'ayant précocement identifiée, la démographie historique française a volontiers euphémisé la mobilité rurale en la qualifiant de "micromobilité » et en l'excluant ainsi du domaine de la réflexion sur les migrations.

28. Les premiers historiens de la famille ont eux aussi distingué des formes de ménages hétérogènes. Mais ils ont rattaché familles nucléaires, familles souches ou familles étendues à des ensembles anthropologico-culturels macroscopiques disjoints : adeptes d'une perspective macroscopique, ils ont par exemple divisé la France ou l'Europe en trois modèles régionaux. Le propre d'une étude micro est de relativiser ces segmentations générales : l'intérêt d'un suivi « au ras du sol » est de montrer que toutes choses égales d'ailleurs (c'est-à-dire à village et à position sociale donnés), l'hétérogénéité des formes familiales est aussi forte au niveau local qu'à l'échelle d'une macrorégion. 
sociales. En amont, la clé de la diversité observée se situe dans les modes d'accès des lignées aux ressources locales : qu'il s'agisse, par exemple, d'orientations professionnelles ou de destinations migratoires, certaines lignées privilégient la circulation interne des ressources et des informations, tandis que d'autres ont des liens plus éclatés qui donnent un ancrage environnemental spécifique à chacun de leurs membres. Aucune forme familiale ne peut dès lors prétendre à une quelconque supériorité intrinsèque sur une autre : chaque modèle porte sa cohérence, ses coûts et ses avantages $^{29}$.

Par là même éclate l'hypothèse, fondatrice du modèle homéostatique, d'une modalité unique d'ajustement des populations aux ressources: les familles disposent au contraire de plusieurs manières pour tirer parti de leur environnement. L'analyse microscopique ne fait pas pour autant basculer dans l'utopie d'une maîtrise sans limite des individus sur leur destinée : l'organisation familiale, loin d'être décidée in abstracto et dans une logique d'optimisation stratégique, semble plutôt découler d'une chaîne de décisions ponctuelles et limitées, dont la succession seule finit par créer un système, en soi cohérent mais dont il devient difficile de sortir une fois qu'il est constitué ${ }^{30}$.

À l'issue de ce raisonnement et de ces exemples, on comprend le déplacement que provoque l'adoption d'une échelle micro. À l'idée d'une loi d'airain des ressources, qui s'appliquerait de manière indifférenciée à niveau socio-économique donné, se substitue une vision où une structure intermédiaire - la famille — vient décliner les contraintes de l'environnement global. Le départ avec le modèle homéostatique est multiple. Au contraire de ce dernier, la migration n'est plus systématiquement traitée comme une décision "négative », comme un simple exutoire. Elle devient une ressource potentielle offerte aux individus pour élargir les ressources de leurs lignées, ou pour échapper aux tensions internes qui s'y produisent. La prise en compte d'un cadre familial étendu amène à raisonner en termes de «portefeuille spatial », véritable patrimoine collectif que la lignée met plus ou moins en commun selon son mode d'organisation. Non définissable a priori, l'espace pertinent devient un espace immanent, dont les contours sont propres à chaque groupe familial, et observables seulement ex post, via les comportements de ses membres. Extension spatiale mais aussi temporelle : la détermination des actions n'est plus réductible à un stimulus du moment, elle devient le fruit d'une histoire souvent longue, d'une chronologie propre à chaque lignée.

L'ensemble de ces propositions ouvrent autant de pistes de recherches, et de manières de réinterroger les sources. Reste comme toujours à en exami-

29. La dichotomie est formulable dans les termes de Granovetter, 1973.

30. C'est du moins ce qui ressort de Hontebeyrie et Rosental, 1999. 
ner le coût, c'est-à-dire à s'interroger sur les limites heuristiques de l'échelle micro.

MICRO OU LOCAL ?

L'une des critiques les plus fréquemment formulées à l'encontre des analyses micro concerne à n'en pas douter leur caractère censément trop singulier. Sorties du village de Santena, que valent les observations de Levi sur les stratégies des métayers? Sur la question spécifique du modèle homéostatique, l'historien allemand Jürgen Schlumbohm se heurte à la même aporie. La microhistoire qu'il propose de la petite paroisse de Belm montre que les villageois ne sont pas restés soumis au carcan d'un nombre fini d'exploitations agricoles. Bien au contraire, ils ont su au cours des $\mathrm{XVIII}^{\mathrm{e}}$ et $\mathrm{XIX}^{\mathrm{e}}$ siècles élargir leurs gammes de ressources et diversifier leurs activités à l'intérieur ou hors du village. Leur population, du même coup, est sortie de la trappe homéostatique et n'a cessé de croître ${ }^{31}$. Schlumbohm, malgré des différences de méthode, se réclame lui aussi de la microhistoire, dont il incarne une autre version nationale. Sa perspective n'est pas tout à fait la même que celle des transalpins : il s'agit moins de reconstituer les attendus des actions individuelles ou familiales et le poids de l'incertitude auxquelles elles sont soumises, que de raisonner à l'échelle — locale mais au fond macroscopique — d'une petite communauté rurale. Par maints aspects, il partage avec les Italiens une même définition des enjeux de son approche : son ambition n'est pas de dresser un tableau représentatif d'un ensemble géographique plus étendu, mais de réexaminer un modèle à vocation générale. Dans un cas comme dans l'autre, l'histoire se pense et se pose comme une science sociale, dont l'objet dépasse le terrain d'observation pour se placer au niveau de la théorie sociale. Il reste qu'une différence de tonalité les sépare, qui montre si besoin est les multiples usages et invocations que l'on peut faire du micro.

Levi manifeste le souci, qui n'est pas sans résonances mertoniennes, d'identifier des formes d'organisation sociale qui, sans être nécessairement universelles, peuvent aider à éclairer d'autres situations, ou au minimum à élargir la gamme des problématiques que l'on peut leur soumettre. Schlumbohm, au contraire, continue à se situer par rapport à un modèle qui préexiste à son approche. En invalidant le modèle homéostatique sur un cas précis, il laisse ouverte la question des leçons à tirer de son analyse : soit le

31. Schlumbohm, 1996. 
cas de Belm est unique, soit il indique qu'à travers des modalités locales éventuellement différentes chaque paroisse parvient à s'affranchir du cadre homéostatique. Dans ce dernier cas, se pose la question de la nature du savoir à produire : doit-on reformuler les relations entre populations et ressources de manière alternative au modèle homéostatique, ou renoncer à l'idée même de modèle?

La question que soulève l'échelle micro n'est donc pas sa capacité à déboucher sur des propositions généralisables. Négativement, le reproche de ne pas produire des résultats représentatifs est anachronique par rapport à ses fondements : quoique souvent intériorisé comme une évidence, ce souci renvoie à un univers épistémologique situé, façonné par l'histoire quantitative des décennies d'après-guerre et par l'empreinte de la statistique administrative qui pesait sur elle. Positivement, si les approches micro, via des modalités variées, se donnent pour objet l'identification de mécanismes situés d'engendrement du social, il est indéniable qu'elles sont dotées d'une valeur problématique générale, c'est-à-dire transposable à d'autres terrains. Aussi la véritable question que pose l'adoption d'une perspective micro ne porte-t-elle pas sur la portée mais sur la nature du savoir qu'elle produit. Cette imprécision — pour ne pas dire cet impensé — est sans doute responsable des usages contradictoires du micro : celui-ci peut porter des contenus très différents, dont le plus minimaliste est une espèce de vague sensibilité au contingent, et dont le plus ambigu repose sur une confusion entre microscopique et local.

Ce dernier amalgame est sans doute le plus fréquent. Plutôt que de le théoriser excessivement, revenons-en à la matérialisation commode que fournit l'exemple, précédemment évoqué, des parcours de mobilité dans la France du XIX ${ }^{\mathrm{e}}$ siècle. Ce cas repose tout d'abord sur un échantillon national : il confirme que ce n'est pas un parti pris théorique, mais un problème de sources, qui restreint d'ordinaire le micro au local. Pour peu que l'on dispose de moyens plus importants ${ }^{32}$, il est possible de pratiquer la microanalyse... à une vaste échelle. C'est avant tout la lourdeur d'un dépouillement intensif qui, selon leurs modalités particulières, a poussé la microhistoire, qu'elle soit italienne, allemande ou anglaise ${ }^{33}$, à privilégier une espèce d'histoire totale réduite au niveau de la paroisse ${ }^{34}$. Des approches qui, à des degrés divers, prétendent se situer au ras du sol pour reformuler les catégories du savoir établi, se heurtent dès lors à un paradoxe : le cadre

32. Tel a été le cas de l'enquête Trois mille familles, déjà mentionnée supra n. 23.

33. Dans le cas italien, le parti pris configurationnel pousse à la reconstitution la plus exhaustive possible des réseaux interpersonnels et, du même coup, à une patiente utilisation des sources nominatives. Dans les cas allemand et, plus encore, anglais, la microhistoire se rapproche d'une espèce d'histoire locale totale saisie dans la longue période, prémisses qui elles aussi limitent l'aire géographique contrôlable. Voir Wrightson et LevinE, 1998.

34. LEVI, 1981. 
même de leur analyse — l'unité administrative locale — est généralement donné d'avance, alors qu'il structure toute la construction de l'objet.

Certes, la recherche amène souvent à relativiser sa pertinence : tandis que Levi identifie les relations qui connectent les notables de Santena à la cour ducale du Piémont, Schlumbohm examine comment les habitants de Belm rayonnent alentour pour diversifier leurs activités. Mais si nous sommes loin du modèle orthodoxe de la monographie paroissiale ${ }^{35}$, il reste que la microanalyse du village ne reformule pas la perception que l'on peut avoir de son environnement et de sa région. En fait, elle est par essence soumise à un paradoxe : en construisant son objet par connexions successives, la microhistoire réfute les découpages et les clôtures a priori qui en sont d'ordinaire proposés. Mais en montrant qu'un objet même minuscule s'insère dans une configuration vaste que son étude permet d'éclairer, elle invalide elle-même l'idéal de systématicité qui la fonde. Ce dilemme, hérité de l'ethnographie qui en a elle-même âprement discuté, révèle les ambiguïtés d'une méthode qui, par maints aspects, repose sur l'illusion toute positiviste de saisir un objet dans sa totalité, voire dans son essence.

L'étude de la mobilité à travers les parcours généalogiques fournit une nouvelle fois une traduction immédiate de ce problème. L'espace, en effet, est segmenté et hiérarchisé. Il est parcouru de divisions et de polarisations invisibles, qu'elles reposent sur des différentiels économiques ${ }^{36}$ ou sur des liens préférentiels d'origine historico-géographique ${ }^{37}$. Ces déclinaisons orientent les flux de mobilité à la manière de courants : faute de les saisir, on ne peut entièrement comprendre les destinations des migrants. Or, si le suivi de trajectoires familiales permet de découvrir des processus inédits, il occulte l'environnement social dans lequel elles se déroulent. Cette formulation pousse, à première vue, à formuler le problème en termes d'échelles. Au micro appartiendrait l'observation fine des trajectoires familiales et des motivations à migrer; au macro, l'analyse des hiérarchies spatiales globales.

L'examen de la littérature semble légitimer cette opposition. Les dénivellations de l'espace national sont volontiers saisies à une échelle large, généralement en France celle des départements pour lesquels sont disponibles les séries de la statistique administrative ${ }^{38}$. L'alternative - constituer des séries statistiques plus fines, en agrégeant les informations disponibles dans les sources nominatives — pose le même problème que précédemment, celui de la lourdeur des dépouillements. En pratique, il est

35. Qui, dans la lignée d'une longue tradition, trouve sans doute sa formalisation la plus précise in Gautier et Henry, 1958.

36. Goreux, 1956, et Sicsic, 1991.

37. Hägerstrand, 1957.

38. LePETIT, 1986. 
difficile de dépasser l'échelle de la paroisse ou de la commune, mais il s'agit là d'une contrainte empirique et non d'une nécessité liée à l'objet.

Aussi doit-on, là encore, renoncer à une rigidification théorique de l'opposition entre échelles micro- et macroscopique, pour raisonner en termes d'usage des sources. Dans ses versions italienne ou allemande, la microhistoire prolonge une pente entamée au milieu du $\mathrm{xx}^{\mathrm{e}}$ siècle, à savoir la promotion des sources nominatives (état civil, listes d'habitants, documents fiscaux et patrimoniaux) comme matériau de base de l'histoire sociale quantitative. Prolongement, mais aussi inflexion : dans les décennies d'après-guerre, ces sources sont avant tout un palliatif à l'absence de séries statistiques considérées comme fiables, et une matière première permettant d'en constituer ex nihilo ${ }^{39}$. À partir des années soixante-dix, la dimension quasi biographique de ces documents commence à être prise au sérieux : à l'anonymat des grands nombres on substitue la diversité des comportements, en ne les agrégeant plus à l'échelle de la population mais à celle des ménages ou des lignées. Mais ce changement de regard, qui est une des manières de caractériser la sensibilité « micro », accentue encore la lourdeur des dépouillements, dénoncée comme une contrainte dès la fin des années cinquante $^{40}$ : malgré le déplacement des problématiques, il encourage plus que jamais à privilégier l'étude de petites entités spatiales ou sociales, de préférence parcourues de liens relativement denses pour légitimer la clôture de l'objet. En somme, l'équation (même lâche), entre un type de sources, d'outils, d'échelle et de déterminations préférentielles, est en grande partie le produit d'une contrainte archivistique. Il n'est pas accidentel qu'elle se renforce dans une décennie, les années soixante-dix, qui renonce progressivement aux grandes enquêtes collectives d'histoire sociale fondées sur une forte division sociale du travail savant et sur une main d'œuvre abondante dans une période de forts recrutements.

Cette relecture qui, tout en corroborant les fondements intellectuels du passage au micro, en réhabilite les aspects matériels et, du même coup, en relativise la singularité, est-elle testable? Deux recherches contemporaines, consacrées au modèle homéostatique, donneront ici un début de réponse. La première part d'une mise à l'épreuve explicite des déterminations micro : reprenant le fil rouge de cet article, elle consiste à compléter la base de données généalogique déjà mentionnée sur la France du XIX ${ }^{\mathrm{e}}$ siècle, et à l'insérer dans un tissu de déterminations macroscopiques possibles. Il s'agit concrètement de rapporter les trajectoires des individus non seulement à leurs attributs propres (alphabétisation, fortune, etc.) ou à leurs liens interpersonnels, mais aussi aux ressources plus larges de leur environne-

39. Rosental, 1996.

40. BAEHREL, 1957. 
ment. Tout est encore une fois affaire d'archives : il s'agit d' « inventer » de nouvelles sources, en puisant dans le prodigieux inventaire communal réalisé en France au XIX ${ }^{\mathrm{e}}$ siècle. Enquêtes administratives ou économiques, guides de tourisme, dictionnaires de communes ont amoncelé et souvent publié des dizaines de données sur chaque commune française. Elles confirment la distinction formulée précédemment en permettant de disposer d'informations macroscopiques mais locales (et très fines): salaires moyens communaux mais aussi distance à la plus proche gare ou école, volume de courrier envoyé et reçu pour s'en tenir à quelques exemples ${ }^{41}$.

Cette précision permet de confronter des déterminations multiples : propriétés sociales, configurations interpersonnelles, environnement économique immédiat, mais aussi richesse des infrastructures collectives. À chacune correspond implicitement un type d'explication sociologique. Les transformations statistiques des dernières décennies, avec la diffusion de l'économétrie dans les sciences sociales, permettent de donner une traduction littérale à cette notion de « confrontation » : il est possible de comparer des registres explicatifs qui d'ordinaire sont incommensurables, ou que l'on se contente d'accumuler sans tenter de les hiérarchiser et de les articuler $^{42}$. Il y a là matière à situer à leur place respective, et avec leurs poids relatifs, les raisons alléguées d'ordinaire pour rendre compte des migrations.

Les résultats de l'approche ne sont pas plus aisément classables que ses attendus. Des formulations habituelles du modèle homéostatique, ils retiennent l'importance des différentiels économiques dans l'orientation de la mobilité, mais en les saisissant à une échelle fine, et en les étendant au monde rural : d'un village à l'autre, les ressources globales diffèrent et orientent les flux de mobilité, les villes n'ayant pas le monopole des facteurs attractifs. En même temps, la vision passive du modèle homéostatique est battue en brèche. Qu'il s'agisse du type d'organisation des familles ou de l'intégration dans des canaux d'échanges (mesurés par les volumes de courriers), le mode d'insertion à l'environnement module la propension à migrer. De même, l'économisme strict est remis en cause par la mise en évidence de l'effet des infrastructures publiques, produit en dernière instance des compétitions entre communes pour se partager les investissements collectifs. Au total, la vision d'une masse paysanne soumise à l'ajustement mécanique aux ressources s'estompe au profit d'une vision plus dynamique, mais aussi plus conflictuelle, qui privilégie l'affrontement entre des formes différentes d'accès à l'environnement local et régional, tant à l'échelon des lignées qu'à celui des communes. D'ordinaire, la cri-

41. Bourdieu et al., 2000.

42. Selon les critiques adressées à l'historiographie des migrations par Akerman, 1975, p. 12. 
tique des explications fonctionnalistes passe par une relecture micro des grands modèles historiographiques. Dans le cas présent, elle articule le micro dans une gamme plus large de déterminations et brise son équation avec le cadre local.

MICROHISTOIRE ET MÉGAHISTOIRE

Cette relativisation trouve un écho inattendu dans une seconde recherche contemporaine, qui s'inscrit dans le cadre historiographique a priori radicalement opposé de la world history. Forgée aux États-Unis, celle-ci encourage les comparaisons ambitieuses - elles traversent continents et civilisations - , afin de mettre à l'épreuve des notions et des schémas qui, tout en se revendiquant volontiers comme universels, donnent implicitement ou explicitement à l'Europe - voire à l'Europe occidentale — une valeur de référence. Dans un champ d'investigation potentiellement sans limites, la world history s'est intéressée, entre autres, au modèle homéostatique.

À première vue, sa saisie du problème ne peut être qu'orthogonale aux révisions que nous avons décrites. La world history, à mille lieues du micro, développe une sorte de vision post-braudélienne dans laquelle l'unité d'observation est le continent ou l'Empire. Concernant les modèles d'équilibre population/ressources, elle s'est ainsi attelée à une comparaison entre les histoires démographiques européenne et chinoise, pour montrer que cette dernière est moins «exceptionnelle» et surtout moins passive qu'on ne l'a longtemps cru $^{43}$. Est mise en cause la dichotomie entre les deux mécanismes homéostatiques, la régulation par le mariage censée avoir caractérisé l'Occident, la régulation par la mortalité supposée avoir été le lot d'une population chinoise à l'évolution débridée ${ }^{44}$. Il en ressort un modèle dans lequel l'ancienne Chine, par son organisation culturelle et politique, parvient à infléchir l'effet attendu de déterminations démoéconomiques que l'on aurait pu juger irréversibles. Des phénomènes sociaux comme l'infanticide des filles, ou politiques comme les actions de l'État en matière d'irrigation, de prix des grains, de colonisation de terres, se révèlent ici comme des facteurs décisifs pour l'évolution de la population. L'apport des auteurs ne réside certes pas dans l'identification de ces

43. Voir, en part., Lavely et Wong, 1998.

44. Chez Malthus, l'opposition entre l'Europe et la Chine est polaire, les Chinois incarnant notamment une population censée vivre à peine au-dessus du niveau de subsistance. Voir l'analyse de WrigLEY, 1986, p. 55 sq. 
paramètres dont beaucoup sont connus de longue date, mais dans la tentative de les intégrer comme endogènes dans une formalisation démographique qui, à l'arrivée, réduit la distance supposée entre les mécanismes régulateurs européens et chinois.

Il est intéressant d'observer que tout en procédant à une échelle beaucoup plus vaste, et tout en mettant l'accent sur des facteurs (culturels ou politiques) spécifiques ${ }^{45}$, cette confrontation eurasienne débouche sur des conclusions convergentes avec celles qui sont issues des approches micro. Dans un cas comme dans l'autre, on est amené à remettre en cause un modèle trop mécanique, et enfermé dans une définition trop restrictive des frontières de l'économie ou de la démographie. Il s'en faut de beaucoup que cette convergence soit sans précédent. Ester Boserup, dans un ouvrage devenu classique ${ }^{46}$, avait montré que les populations sont susceptibles de répondre au piège malthusien par l'innovation technologique, desserrant ainsi les contraintes de l'environnement. Elle procédait de manière globale, montrant implicitement que le couple micro/macro ne correspond pas par essence à une opposition entre réaction et contrainte.

Mais, dans le cas présent, le parallèle est d'autant plus saisissant qu'il est renforcé par ses attendus archivistiques. La relecture du cas chinois opère en réalité par réinterprétation de fragments. Ce sont des études locales dispersées, souvent menées au hasard des sources anciennes disponibles (dépendant elles-mêmes du statut institutionnel des populations considérées), qui fondent l'essentiel de l'analyse. Les données « macro » — issues de computations sur la population de l'Empire vieilles parfois de plusieurs siècles - sont, en effet, pour la plupart suspectes aux yeux des auteurs. C'est surtout l'histoire de leur production qui entre dans leur argumentation: les jugements catastrophistes qui ont longtemps été portés sur la démographie chinoise sont pour partie attribués à l'irrégularité de ces données, elles-mêmes produits d'un contrôle étatique inégalement efficace selon les époques. Il en résulte un intéressant paradoxe, à savoir la construction d'une démonstration continentale assise en dernier ressort sur une poignée d'études locales. Situation d'autant plus frappante que des pays, comme la France ou l'Angleterre, où la démographie historique a permis, via la réalisation d'enquêtes ad hoc, de disposer de données à l'échelle nationale, n'ont pas été épargnés par les réévaluations micro.

Ce contraste reprend une observation que l'on a retrouvée tout au long de cet article : lorsque l'on entre dans le détail, c'est-à-dire au-delà de

45. L'accent mis sur le rôle des politiques de population n'est pas en soi sans précédent : là encore, les raisonnements homéostatiques ont été suffisamment plastiques pour les intégrer de manière plus ou moins formalisée et plus ou moins extrême, voir EHMER, 2000. Malthus luimême prend en considération cette dimension politique, ainsi plus généralement que les préférences «culturelles » des populations, voir WRIGLEY, 1986.

46. Boserup, 1965. 
l'immédiateté de la méthode pour ne pas dire du choix de terrain, l'opposition entre micro et macro devient souvent aussi problématique que l'était l'équation entre micro et local. Dans le cas présent, la dichotomie microhistoire/world history (celle-ci étant une forme extrême de macrohistoire, voire de mégahistoire) concerne surtout des stratégies narratives différentes. Est-ce à dire que les étiquetages historiographiques sont des leurres? Ce serait oublier que leurs revendications sont chaque fois historiquement situées et incorporent des a priori théoriques pas toujours explicités.

L'un des fondements de la microstoria est le rejet par ses fondateurs des approches idéalistes en histoire. Mais l'alternative retenue, reconstituer son objet à partir d'une posture d'ignorance en faisant du réseau interpersonnel un déterminant majeur des comportements, repose sur un coup de force théorique : prétendre échapper aux préconstructions catégorielles, tout en s'appuyant sur la construction théorique de la sociologie configurationnelle ${ }^{47}$. Cette hypostasie du lien conditionne, par circularité, le registre des explications produites, même lorsqu'elles se réclament d'une pure légitimité empirique. Par contraste, la world history est issue d'une réflexion sur le multiculturalisme dont les fondements sociologiques sont immédiats : dans un pays qui attire migrants et étudiants du monde entier, comment élaborer une histoire affranchie de tout européocentrisme? Ainsi, le système de preuves décrit à propos de la Chine est le produit d'un parti pris comparatif qui, prenant sens à l'échelle transcontinentale, rendrait peu efficace une rhétorique « micro ». Et l'attention portée à la construction politique de la population provient de ce qui fut la première cible de la world history, à savoir l'État, objet pour lequel les sciences sociales ont longtemps multiplié les catégorisations continentales et donné la part belle à l'Occident.

Ces indéniables nuances de sensibilité historiographique, et les effets de savoir qu'elles induisent, sont moins réductibles à une systématisation abstraite que des oppositions théoriques tranchées comme celle qui oppose micro et macro. Mais dans un domaine où des contingences aussi déterminantes que l'accessibilité des sources créent des situations nationales incommensurables, c'est plus encore qu'à l'accoutumée à des points de vue situés et fortement contraints qu'il convient de rapporter les manières de faire des historiens. L'étiquette micro pour microstoria a rencontré un vif succès en France mais ne rend pas justice de la diversité des autres microhistoires nationales, ni des convergences inattendues avec ce que l'on pourrait appeler la mégahistoire. D'un côté le microscopique, que peuvent investir les contenus les plus hétérogènes; de l'autre la microstoria, boîte à

47. Qu'elle passe par Simmel, Elias ou l'école d'anthropologie sociale de Manchester. Voir l'explicitation de GrENDI, 1982. 
outils ingénieuse issue de l'historiographie italienne des années soixantedix : historiographiquement comme heuristiquement, on ne peut que gagner à ne pas confondre les deux termes dans une même équation.

Paul-André Rosental

(août 2000). 


\section{LISTE DES RÉFÉRENCES}

Akerman (Sune), 1975, From Stockholm to San Francisco. The development of the historical study of external migrations, Uppsala, Almqvist et Wiksell.

BAEHREL (René), 1957, «La mortalité sous l'Ancien Régime. Remarques inquiètes », Annales. Économies, sociétés, civilisations, vol. XII, 1, p. 85-98.

Boserup (Ester), 1965, The Conditions of agricultural growth. The economics of agrarian change under population pressure, Londres, G. Allen \& Unwin.

Bourdieu (Jérôme), Postel-Vinay (Gilles), Rosental (Paul-André) et Suwa-EisenMANN (Akiko), 2000, « Migrations et transmissions inter-générationnelles dans la France du $\mathrm{XIX}^{\mathrm{e}}$ et du début du $\mathrm{Xx}^{\mathrm{e}}$ siècle », Annales. Histoire, sciences sociales, $55^{\mathrm{e}}$ année, 4 , p. 749-790.

Coleman (David), 1986, «Population regulation. A long-range view », in Coleman et SCHOFIELD, éd., p. 14-41.

Coleman (David) et Schofield (Roger S.), éd., 1986, The State of population theory. Forward from Malthus, New York, NY, Basil Blackwell.

Corbin (Alain), 1998, Le Monde retrouvé de Louis-François Pinagot. Sur les traces d'un inconnu, 1798-1876, Paris, Flammarion.

DuPÂQUIER (Jacques), 1972, « De l'animal à l'homme. Le mécanisme autorégulateur des populations traditionnelles », Revue de l'Institut de sociologie, 2, p. 177211.

DupÂQuiER (Jacques) et KessLER (Denis), éd., 1992, La Société française au XIX siècle, Paris, Fayard.

EHMER (Josef), 2000, « German origins of the homeostatic model. Gunther Ipsen's and Gerhard Mackenroth's historical-sociological population theories », $3^{\text {rd }}$ Social Science History Conference, Amsterdam, mimeo.

FARGe (Arlette) et Revel (Jacques), 1988, Logiques de la foule. L'affaire des enlèvements d'enfants, Paris 1750, Paris, Hachette.

FERTIG (Georg), 1998, «Balancing, networking and the causes of emigration. Early German transatlantic migration in a local perspective, 1700-1754 », Continuity and Change, vol. XIII, 3, p. 419-442.

Gautier (Étienne) et Henry (Louis), 1958, La Population de Crulai, paroisse normande. Étude historique, Paris, Presses universitaires de France.

Goreux (L. M.), 1956, «Les migrations agricoles en France », Études et conjoncture, vol. X, p. 327-376.

Granovetter (Mark S.), 1973, « The strength of weak ties », American Journal of Sociology, vol. LXXVII, 6, p. 1360-1380.

GRENDI (Edoardo), 1982, « Norbert Elias. Storiografia e teoria sociale », Quaderni Storici, vol. L, 2, p. 728-739.

Hägerstrand (Torsten), 1957, « Migration and area. Survey of a sample of Swedish migration fields and hypothetical considerations on their genesis », in David Hannerberg, Torsten Hägerstrand et B. Odeving, éd., Migration in Sweden. A symposium, Lund, Cwk Gleerup, p. 27-158.

Hontebeyrie (Juliette) et Rosental (Paul-André), 1999, Les Lieux et les liens. Parenté, transmissions et territoires dans un faubourg lillois (Loos-lès-Lille, 1840-1960), Paris, rapport présenté à la mission du Patrimoine ethnologique.

Langley (Leroy L.), éd., 1973, Homeostasis. Origins of the concept, Stroudsburg, PA, Dowden, Hutchinson \& Ross. 
Laslett (Peter) et Wall (Richard), éd., 1972, Household and family in past time, Cambridge, Cambridge University Press.

Lavely (William) et Wong (R. Bin), 1998, « Revising the Malthusian narrative. The comparative study of population dynamics in late Imperial China », Journal of Asian Studies, vol. LVII, 3, p. 714-748.

LEE (Ronald D.), 1978, « Models of preindustrial population dynamics with application to England », in Charles Tilly, éd., Historical studies of changing fertility, Princeton, Princeton University Press, p. 155-207.

LEE (R. D.), 1990, «The demographic response to economic crisis in historical and contemporary populations ", Population Bulletin of the United Nations, 29 , p. $1-15$.

LEPETIT (Bernard), 1986, « Sur les dénivellations de l'espace économique en France dans les années $1830 »$, Annales. Économies, sociétés, civilisations, 6, p. 12431272.

LEPETIT (B.), 1993, «Architecture, géographie, histoire. Usages de l'échelle », Genèses, 13, p. 118-138.

LeVI (Giovanni), 1981, «Villaggi », Quaderni Storici, vol. XLVI, 1, p. 7-10.

Levi (G.), 1989, Le Pouvoir au village. Histoire d'un exorciste dans le Piémont du XVII ${ }^{e}$ siècle, Paris, Gallimard.

MiLo (Daniel), 1991, " Pour une histoire expérimentale ou le gai savoir », in Daniel Milo et Alain Boureau, éd., Alter-histoire. Essais d'histoire expérimentale, Paris, Les Belles Lettres, p. 9-55.

Revel (Jacques), 1989, «L'histoire au ras du sol », préf. à LeVI, 1989, p. I-XXXIII.

Revel (J.), éd., 1996, Jeux d'échelles. La microanalyse à l'expérience, Paris, Seuil.

Rosental (Paul-André), 1996, « Treize ans de débats. De l'histoire des populations à la démographie historique française (1945-1958) », Population, 6, p. 12111238.

Rosental (P.-A.), 1999, Les Sentiers invisibles. Espace, familles et migrations dans la France du XIX siècle, Paris, École des hautes études en sciences sociales.

Schlumbonm (Jürgen), 1996, « Micro-history and the macro-models of the European demographic system in pre-industrial times. Life course patterns in the parish of Belm (Northwest Germany), seventeenth to nineteenth centuries », The History of the Family, vol. I, 1, p. 81-96.

Schweber (Silvan S.), 1993, "Physics, community and the crisis in physical theory », Physics Today, 11, p. 34-40.

Scotт (Rebecca J.), «Reclaiming Gregoria's mule. The meanings of freedom in the Arimao and Caunao valleys, Cienfuegos, Cuba, 1880-1899», Past and Present, à paraître.

Sicsic (Pierre), 1991, Labor markets and establishment size in nineteenth century France, Ph. D., Cambridge, MA, Harvard University.

STONE (Lawrence), 1980, « Retour au récit ou réflexions sur une nouvelle vieille histoire », $1^{\mathrm{re}}$ éd. 1979, ici Le Débat, 4, p. 116-142.

«Tentons l'expérience», 1989, Annales. Économies, sociétés, civilisations, 6, p. 1317-1323.

The Territorial Mobility of population. Rethinking its forms and functions, 1979, Liège, International Union for the Scientific Study of Population (Papers 13).

Wrightson (Keith) et Levine (David), 1998, Poverty and piety in an English village. Terling, 1525-1700, $1^{\text {re }}$ éd. 1995, ici Oxford, Clarendon Press.

Wrigley (Edward Anthony), 1986, «Elegance and experience. Malthus at the bar of history », in Coleman et Schofield, éd., 1986, p. 46-64.

Wrigley (Edward Anthony) et SchofIeld (Roger S.), 1981, The Population History of England, 1541-1871, Cambridge, Cambridge University Press. 